\section{Orange bags for clinical waste}

With some confusion as to what's involved with the efficient disposal of clinical waste in orange bags, here are some of the most frequently asked questions, answered by Initial Medical, experts in waste management:

Q1: What waste should go in orange bags?

A: Orange 'clinical and infectious waste' is any waste arising from healthcare activities that could pose a risk to public health or the environment, unless properly disposed of. This includes contaminated swabs, gloves, paper towels, dressings, aprons and masks.

Q2: Do you have to put your postcode, contract number and signature on orange bags?

A: All waste bags and containers should be marked with your business name and postcode. All hazardous waste requires a Hazardous Waste Consignment Note - HWCN - which includes information on your practice and its premises code, details of the waste contents and quantity, as well as its packaging. There is no need to sign the bags themselves, as your signature will be required by your service technician when they collect the waste bags for onward disposal.

Q3: How is waste in orange bags disposed of?

A: Orange waste may be treated to render it safe prior to disposal. Alternatively, it can be incinerated.

For more advice on best practice protocols in waste management and segregation, contact Initial Medical today. Visit www.initial.co.uk/ medical or telephone 08708504045 .

\title{
Ultimate versatility in one dental unit
}

For a smart, practical solution in a dental practice with a team of practitioners look no further than the Puma ELI Ambidextrous from Castellini.

The Puma ELI Ambidextrous is compact and adaptable and offers superior manoeuvrability to optimise the workspace. As its name suggests, this dental unit provides 'ambidextrous' style and elegance, which can be set up for a wide range of operating positions quickly and easily.

The clinician's set up can be changed from left to right or vice versa in minutes. Instruments are arranged both ergonomically and with optimised vertical excursion to allow each user to bring the instruments to the most convenient height.

As well as the cutting-edge technology you would expect from Castellini, the Puma ELI Ambidextrous offers freedom of movement and flexibility for all treatment options. And for convenience, there are four programmable settings so that practitioners can select their preferred working position at the touch of a button.

For a multipurpose dental unit with fast adaptability and efficiency, contact the Castellini team today.

Visit www.rpadental.net. For London and Manchester Sales and Service Centres call 08000933975.

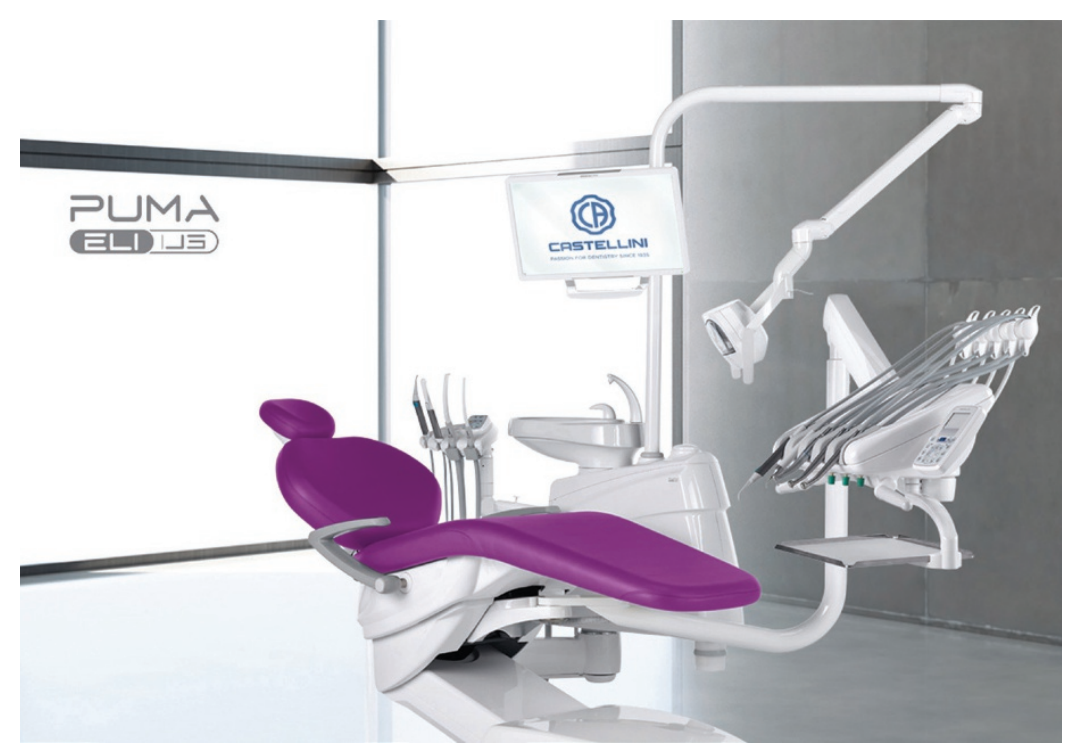

\section{Low stickiness for easy placement}

For a solution that combines speed, strength and optimum handling, Ketac Universal glass ionomer restorative from $3 \mathrm{M}$ Oral Care is the only choice.

With fewer clinical steps, chair time is reduced so patients can enjoy an efficient treatment.

But speed does not mean compromising on performance, because Ketac Universal is proven strong enough for long-term use, including on stress-bearing restorations.

Handling is a dream too, with low stickiness for easy placement.

Available in six shades and with a 24-month continuous fluoride release, find out more about the Ketac Universal glass ionomer restorative by speaking with the experts at $3 \mathrm{M}$ Oral Care today.

For more information, call 08456025094 or visit www.3Mespe.co.uk.

$3 \mathrm{M}$, Ketac and Aplicap are trademarks of the 3M Company.

\section{Superior soft tissue management}

Do you want to achieve excellent aesthetics with dental implants? Of course you do - which is why you should consider the TBR Zlimplant from Dental Express, a trading division of Surgery Express LLP and exclusive UK supplier of TBR products.

The TBR Z1 implant is unique in its zirconia collar-titanium body construction, which, thanks to the biocompatibility of the zirconia collar, allows the implant to be placed at tissue level for excellent soft tissue management and epithelial healing. It also helps reduce the risk of peri-implantitis, which can severely affect the aesthetic future of any dental implant - not to mention function and stability.

To find out more about TBR implants from Dental Express, do not hesitate to contact the highly experienced National Sales Manager, Russel Diffenthal at russel.dif-

fenthal@dental-express.co.uk.To learn more about the $\mathrm{Z} 1 \mathrm{implant}$ visit http://zlimplants.co.uk/.

For more info, visit www.dentalexpress.co.uk, call 08007076212 or email sales@dental-express.co.uk. 